\title{
The five-repetition sit-to-stand test: evaluation of a simple and objective tool for the assessment of degenerative pathologies of the lumbar spine
}

\author{
Victor E. Staartjes, BMed, ${ }^{1,2}$ and Marc L. Schröder, MD, PhD'1 \\ 'Department of Neurosurgery, Bergman Clinics, Amsterdam, The Netherlands; and 'Faculty of Medicine, University of Zurich, \\ Switzerland
}

OBJECTIVE Recently, objective functional tests have generated interest since they can supplement an objective dimension to clinical assessment. The five-repetition sit-to-stand (5R-STS) test is a quick and objective tool that tests movements frequently used in everyday life. The aim of this prospective study was to evaluate the validity and reliability of the $5 R-S T S$ test in patients with degenerative pathologies of the lumbar spine.

METHODS Patients and healthy volunteers completed the standardized 5R-STS, Roland-Morris Disability Questionnaire (RMDQ), Oswestry Disability Index (ODI), visual analog scale (VAS) for back and leg pain, and EQ-5D for healthrelated quality of life (HRQOL). To assess convergent validity, the 5R-STS test times were correlated with these questionnaires.

RESULTS Overall, 157 patients and 80 volunteers were enrolled. Direct correlation with RMDQ $(r=0.49)$, ODI $(r=$ $0.44)$, and VAS for back pain $(r=0.31)$ and indirect correlation with the EQ-5D index $(r=-0.41)$ were observed $(p<$ 0.001). The 5R-STS test showed no correlation with VAS for leg pain and EQ-5D VAS ( $p>0.05)$. In 119 individuals, the $5 R-S T S$ test demonstrated excellent test-retest reliability with an intraclass correlation coefficient of 0.98 . The upper limit of normal, distinguishing patients with and without objective functional impairment, was identified as 10.35 seconds. A severity stratification classified patients with test times of 10.5-15.2, 15.3-22.0, or greater than 22.0 seconds as having mild, moderate, or severe functional impairment, respectively.

CONCLUSIONS The 5R-STS test is a simple and effective tool to describe objective functional impairment. A patient able to perform the test in 10.4 seconds can be considered to have no relevant objective functional impairment.

Clinical trial registration no.: NCT03303300 (clinicaltrials.gov)

https://thejns.org/doi/abs/10.3171/2018.2.SPINE171416

KEYWORDS sit to stand; objective test; degenerative disc disease; lumbar stenosis; lumbar disc herniation; functional impairment

$\mathrm{V}$ ALIDATED patient-reported outcome measures (PROMs) have been the gold standard for evaluating patients in spine surgery for decades. ${ }^{3,6,21,22}$ Even so, objective tools have recently gained substantial importance. ${ }^{7,11,17}$ Some objective functional tests have been introduced and have even found their way into clinical practice, such as the six-minute walk test, timed up and go (TUG) test, and accelerometer-based tools. ${ }^{9,11}$ Their proposed advantages include quick execution, high repeatability, and straightforward interpretation of test results for patients, while also supplementing an objective dimension to clinical assessment. ${ }^{9}$ Moreover, it has been shown that patients frequently show preference for an objective functional test over questionnaires. ${ }^{15}$ For these reasons, the development, standardization, and validation of objective functional tests as adjuncts to conventional PROMs is essential.

Surgical decision-making is based on clinical history; measures of pain along with impaired function and healthrelated quality of life (HRQOL); neurological status; and radiological evidence of disease. ${ }^{9}$ Commonly used PROMs

ABBREVIATIONS DDD = degenerative disc disease; HRQOL = health-related quality of life; ICC = intraclass correlation coefficient; LDH = lumbar disc herniation; ODI = Oswestry Disability Index; OFI = objective functional impairment; PROM = patient-reported outcome measure; RMDQ = Roland-Morris Disability Questionnaire; TUG = timed up and go; ULN = upper limit of normal; VAS = visual analog scale; ZOI = zone of indifference; 5R-STS = five-repetition sit-to-stand.

SUBMITTED December 21, 2017. ACCEPTED February 8, 2018.

INCLUDE WHEN CITING Published online June 29, 2018; DOI: 10.3171/2018.2.SPINE171416. 
are the visual analog scale (VAS) for pain, Oswestry Disability Index (ODI) and Roland-Morris Disability Questionnaire (RMDQ) for functional impairment, and the EQ-5D for HRQOL. Because accurate measurement of these parameters is critically important in spine surgery, and considering that the surgeon's assessment may considerably diverge from the patient's self-rating, adding an objective dimension to clinical decision-making may prove useful. ${ }^{9,20}$ Furthermore, some patients may present with symptoms, or improvements after treatment, that simply cannot be captured by standardized questionnaires (e.g., painless symptoms like tingling, foot drop, limping). ${ }^{9,12}$ Combined with traditional PROMs, objective functional tests can extract novel and clinically helpful information.

Primary care, outpatient, and inpatient settings often do not allow for the use of objective functional tests like the six-minute walk test owing to restrictions in space, time, and resources. Therefore, alternative options that are simpler to conduct have been sought. Sit-to-stand movements are commonly performed in everyday life, and are an indicator of physical activity, low-back pain, and muscle strength. ${ }^{14,18,23}$

The five-repetition sit-to-stand (5R-STS) test is a quick and convenient standardized test that is clinically useful and validated for various diseases, including chronic obstructive pulmonary disease and Parkinson's disease (Fig. 1). ${ }^{4,14}$ However, the standardized 5R-STS test has not been evaluated in patients with degenerative spinal pathologies. We aim to add a simple and objective tool to the spine surgeon's armamentarium by evaluating the 5R-STS test for lumbar degenerative pathologies. In a prospective study, we assess the test's correlation with validated PROMs and propose an upper limit of normal (ULN) and a severity stratification.

\section{Methods \\ Study Design and Oversight}

Between October and December of 2017, patients were seen at a specialized outpatient spine surgery clinic. In addition, a representative population of healthy volunteers was enrolled as a control group. Convergent validity was assessed by correlating the 5R-STS results with validated PROMs-namely, VAS scores for back and leg pain, ODI, RMDB, and EQ-5D index and VAS scores. ${ }^{6,21,22}$ In addition, we screened a range of demographic baseline variables and propose reference values to facilitate interpretation of the 5R-STS test. Participants filled in the questionnaires right after performing the test. This prospective trial (clinicaltrials.gov identifier: NCT03303300) was approved by the local IRB (Medical Research Ethics Committees United), and was conducted according to the Declaration of Helsinki. Informed consent was obtained from all participants.

\section{Study Population}

All enrolled patients were candidates for surgery, and they were assessed during outpatient consultations. Inclusion criteria were the presence of lumbar disc herniation (LDH), lumbar stenosis, lumbar spondylolisthesis, degenerative disc disease (DDD), or synovial facet cysts, requir- ing surgical treatment. Patients with hip or knee prosthetics and those requiring walking aides were excluded to eliminate these confounders.

The control group comprised healthy individuals of all ages, and individuals were either volunteers or employees of the department. Most volunteers were the patients' partners and thus exhibit comparable demographics. Volunteers disclosing spinal conditions, hip or knee replacements, other lower-extremity-related complaints, or who required walking aides were excluded.

\section{The 5R-STS Test}

The test was performed according to the protocol described by Jones et al. ${ }^{14}$ The participants were asked to sit down on an armless chair of standard height $(48 \mathrm{~cm})$ and with a hard seat, firmly placed against a wall. The participants were instructed to fold their arms across their chest and to keep their feet flat on the ground. Participants were required to wear stable shoes for the test. To become familiarized with the movement, the participants were asked to stand up fully and sit back down again once without using their upper limbs. If assistance was required, or if the maneuver could not be completed, the test was abandoned. Otherwise, the patients were asked to, starting on the command "go," stand up fully and sit down again, landing on the seat firmly, 5 times as fast as possible. Using a stopwatch, we timed the 5 repetitions from the initial command to the completed fifth stand. This time was recorded as the participant's score. If the patient was unable to perform the test in 30 seconds, or not at all, this was noted and the test score was recorded as 30 seconds.

\section{Statistical Analysis}

Data were reported as mean \pm SD for continuous and numbers (percentages) for categorical data. Analyses were carried out using R version 3.4.2 (The R Foundation for Statistical Computing). Groups were compared using Welch's 2-sample t-test and chi-square test. 5R-STS test times were $\log _{10}$ transformed to achieve normal distribution. Pearson correlation was used to assess the correlation between $\log _{10}$-transformed 5R-STS test times and validated questionnaires. A Wilcoxon test was used to identify any learning effect between measurements. Intraclass correlation coefficients (ICCs) for consistency and absolute agreement, along with their 95\% CI and SEM, were used to examine test-retest reliability. The effects of age, sex, weight, height, body mass index (BMI), and underlying pathology were assessed using linear regression. Standard and adjusted $\mathrm{z}$ scores, the ULN, and the corresponding zone of indifference (ZOI) were calculated (Appendix). ${ }^{26}$ A nonparametric severity stratification was created. ${ }^{9,24} \mathrm{~A}$ two-sided $p$ value of $\leq 0.05$ was considered significant.

\section{Results \\ Cohort}

One hundred fifty-seven patients and 80 healthy volunteers were enrolled (Table 1). Compared with the study group, control participants were younger, had a higher rate of smokers, and presented with higher body weight and BMI (all p < 0.05). The HRQOL data were comparable to 


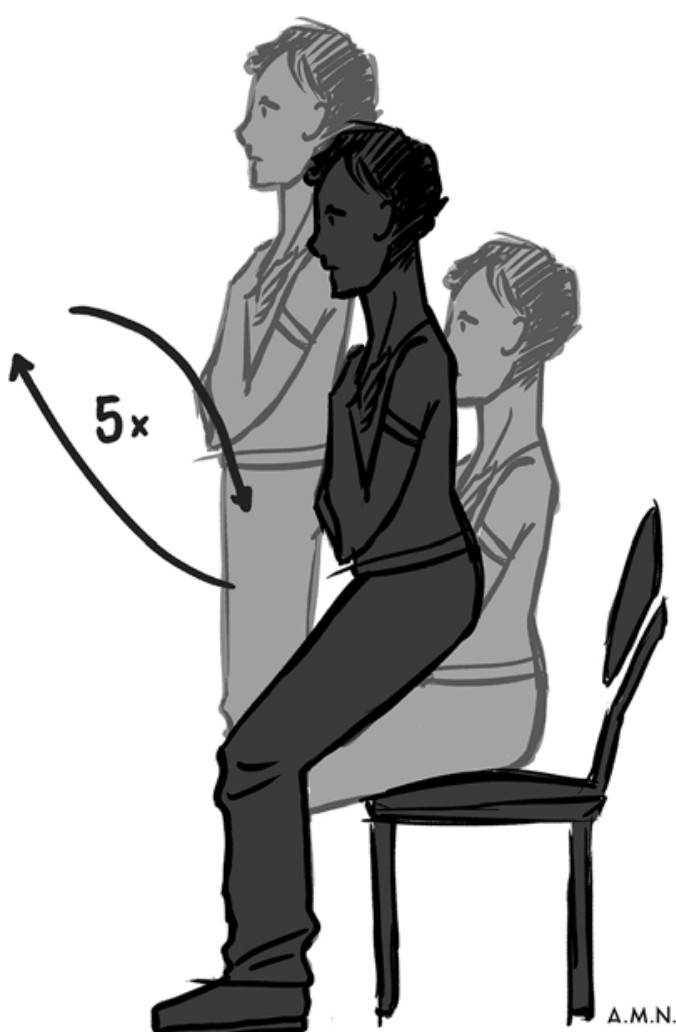

FIG. 1. Scheme of the 5R-STS test. Artist and copyright holder: Anna M. Nikitin. Published with permission.

those of the Dutch population. ${ }^{13}$ Participants in the control group had significantly lower 5R-STS test times (6.44 \pm 1.68 seconds) than those in the study group $(13.32 \pm 7.87$, $\mathrm{p}<0.001)$. Three patients $(2 \%)$ were unable to perform the 5R-STS test independently and abandoned the maneuver.

\section{Convergent Validity}

We observed a direct correlation of logarithmically transformed 5R-STS test times and functional impairment (Fig. 2), as measured by RMDQ ( $\mathrm{r}=0.49,95 \%$ CI $0.36-$ $0.60)$ and ODI ( $r=0.44,95 \%$ CI $0.30-0.56)$, as well as with VAS back pain severity $(\mathrm{r}=0.31,95 \%$ CI $0.16-0.45)$ (all $\mathrm{p}<0.001)$. There was no relevant correlation with VAS leg pain severity ( $\mathrm{p}=0.207$, Fig. 3 ). The $5 \mathrm{R}-\mathrm{STS}$ test also demonstrated indirect correlation with HRQOL (Fig. 4), as measured by EQ-5D index $(\mathrm{r}=-0.41,95 \% \mathrm{CI}-0.53$ to $0.27, \mathrm{p}<0.001)$ but not with EQ-5D VAS score $(\mathrm{p}=0.091)$.

\section{Test-Retest Reliability}

In 66 patients and 53 healthy volunteers, a second 5RSTS test was performed after a 30-minute interval. No learning effect was detected between first (mean $9.35 \mathrm{sec}-$ onds) and second (mean 9.25 seconds) measurements (p $=0.204$ ). The ICC for consistency and for agreement was 0.98 , indicating excellent test-retest reliability according to the Landis-Koch criteria. ${ }^{16}$ Reliability was marginally better in the study group (Table 2). A Bland-Altman plot (Fig. 5) illustrates the test-retest bias of 0.1 seconds, with a $95 \%$ limit of agreement of -2.0 to 2.1 seconds.
TABLE 1. Baseline characteristics of the study and control groups

\begin{tabular}{|c|c|c|c|}
\hline Characteristic & $\begin{array}{l}\text { Study Group } \\
\quad(n=157)\end{array}$ & $\begin{array}{l}\text { Control Group } \\
\qquad(\mathrm{n}=80)\end{array}$ & $\begin{array}{c}p \\
\text { Value }\end{array}$ \\
\hline Male sex & $80(51)$ & $43(54)$ & 0.68 \\
\hline Age, yrs & $49.90 \pm 14.10$ & $43.03 \pm 18.68$ & 0.004 \\
\hline Height, cm & $175.62 \pm 10.44$ & $173.40 \pm 9.29$ & 0.096 \\
\hline Weight, kg & $78.50 \pm 13.51$ & $72.40 \pm 13.58$ & 0.001 \\
\hline $\mathrm{BMI}, \mathrm{kg} / \mathrm{m}^{2}$ & $25.38 \pm 3.28$ & $24.05 \pm 4.25$ & 0.016 \\
\hline Smoking status & & & $<0.001$ \\
\hline Active smoker & $44(28)$ & $14(18)$ & \\
\hline Ceased smoking & $59(38)$ & $17(21)$ & \\
\hline Never smoked & $54(34)$ & $49(61)$ & \\
\hline \multicolumn{4}{|l|}{ Ability to work } \\
\hline Full & $50(32)$ & $76(95)$ & $<0.001$ \\
\hline Limited & $33(21)$ & $2(3)$ & \\
\hline Unable & $74(47)$ & $2(3)$ & \\
\hline Prior spine surgery & $25(16)$ & $6(8)$ & 0.07 \\
\hline History of pain & & & $<0.001$ \\
\hline None-6 wks & $8(5)$ & $73(91)$ & \\
\hline 6 wks- 6 mos & $27(17)$ & $1(1)$ & \\
\hline 6 mos-1 yr & $42(27)$ & $0(0)$ & \\
\hline$>1 \mathrm{yr}$ & $80(51)$ & $6(8)$ & \\
\hline Analgesic drug use & & & $<0.001$ \\
\hline Daily & $120(76)$ & $10(13)$ & \\
\hline Weekly & $15(10)$ & $7(9)$ & \\
\hline Not regularly & $22(14)$ & $63(79)$ & \\
\hline Indication & & & - \\
\hline Disc herniation & $109(69)$ & - & \\
\hline Stenosis & $32(20)$ & - & \\
\hline Spondylolisthesis & $9(6)$ & - & \\
\hline DDD & $5(3)$ & - & \\
\hline Synovial facet cyst & $2(1)$ & & \\
\hline Index level & & & - \\
\hline L2-3 & $4(3)$ & - & \\
\hline L3-4 & $21(13)$ & - & \\
\hline L4-5 & $64(41)$ & - & \\
\hline L5-S1 & $68(43)$ & - & \\
\hline \multicolumn{4}{|l|}{ PROM scores } \\
\hline RMDQ & $11.65 \pm 5.34$ & $0.78 \pm 1.45$ & $<0.001$ \\
\hline ODI & $43.04 \pm 17.60$ & $2.55 \pm 7.39$ & $<0.001$ \\
\hline VAS back pain & $5.83 \pm 2.76$ & $0.95 \pm 1.73$ & $<0.001$ \\
\hline VAS leg pain & $7.35 \pm 1.97$ & $0.40 \pm 1.10$ & $<0.001$ \\
\hline EQ-5D index & $0.41 \pm 0.32$ & $0.94 \pm 0.15$ & $<0.001$ \\
\hline EQ-5D VAS & $51.44 \pm 18.98$ & $86.58 \pm 11.62$ & $<0.001$ \\
\hline
\end{tabular}

$-=$ not applicable.

Continuous variables are presented as mean $\pm S D$ and categorical variables as frequency (percentage).

\section{Upper Limit of Normal}

The ULN was identified as 10.35 seconds with a ZOI ranging between 9.32 and 11.38 seconds. Values in this 

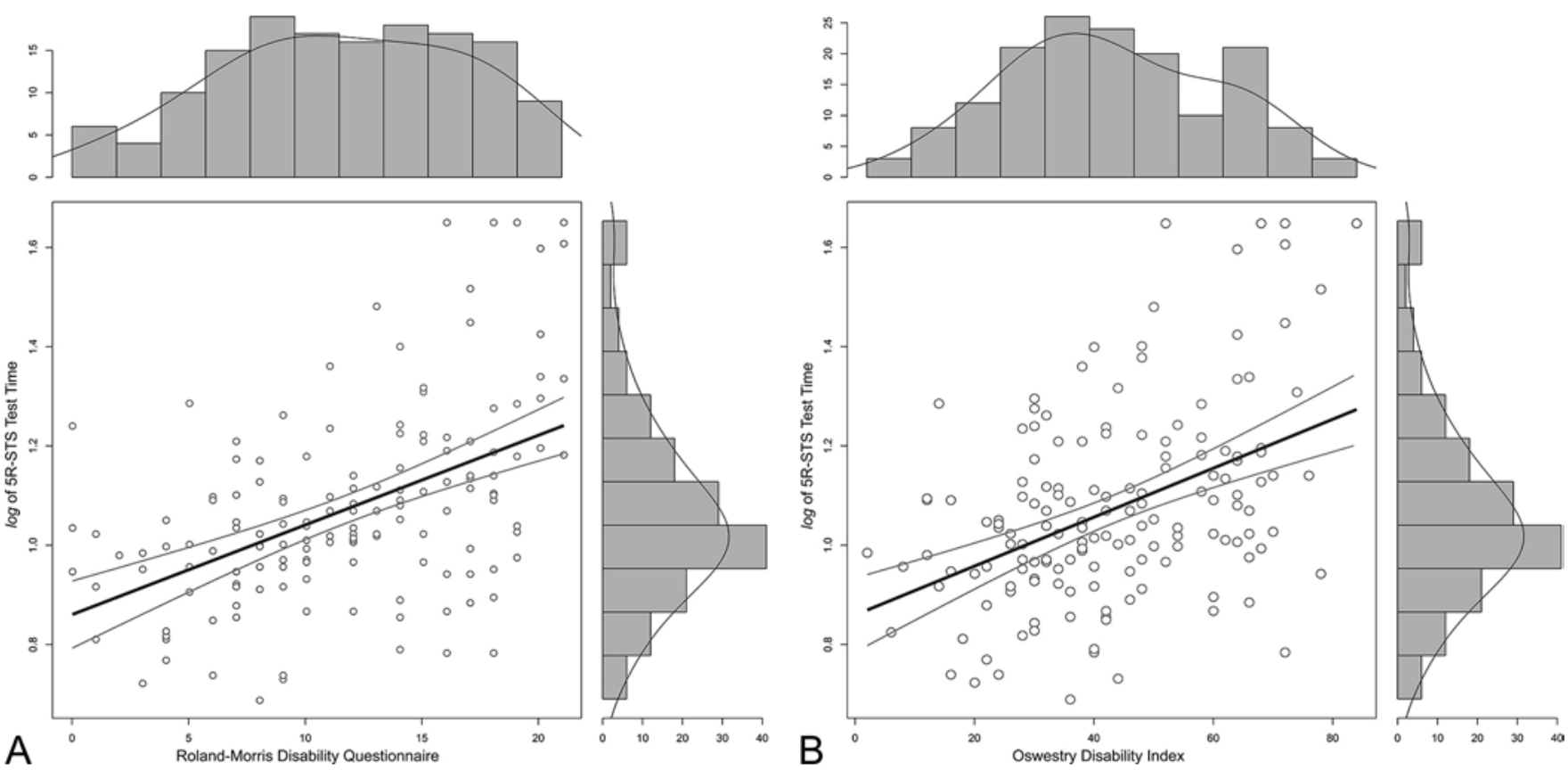

FIG. 2. Scatterplots with marginal histograms showing logarithmically transformed $5 R-S T S$ test times versus measures of functional impairment. There was a direct correlation with $\operatorname{RMDQ}(\mathbf{A})(r=0.49)$ and $\mathrm{ODI}(\mathbf{B})(r=0.44)$ scores.

"gray zone" cannot be unambiguously classified as either healthy or pathological, owing to measurement error.

\section{Severity Stratification}

A severity stratification was developed by partitioning 5R-STS results of the study group into 3 grades of severity in a nonparametric fashion (Table 3). In this cohort, the ULN corresponded roughly to the 50th percentile. Ac-

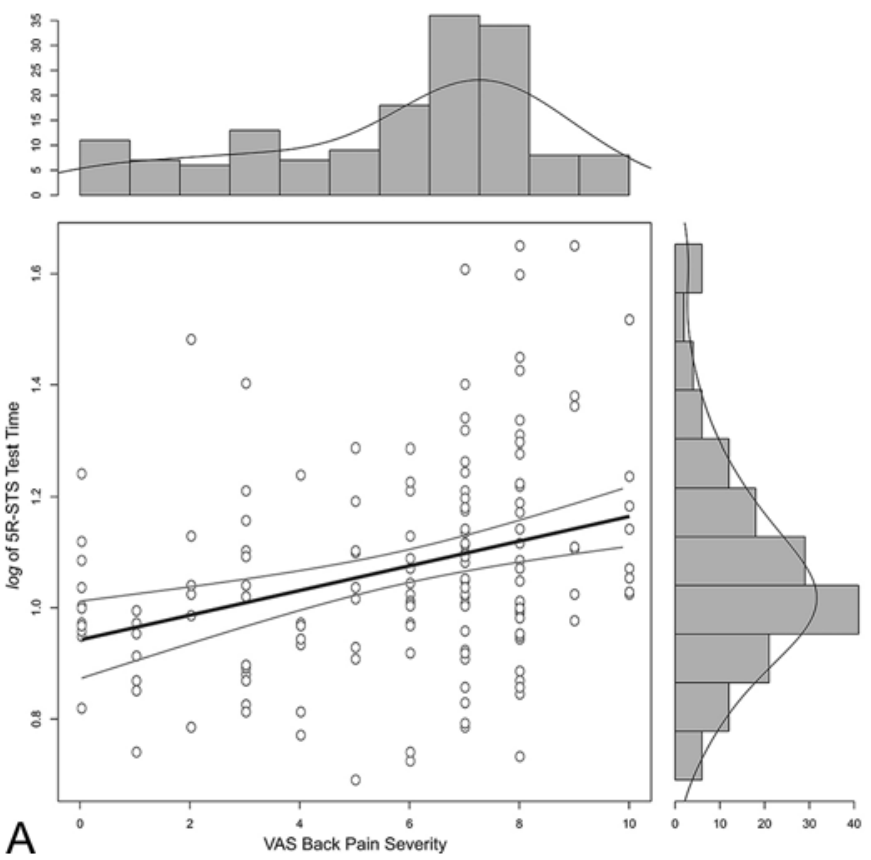

cording to this severity stratification, patients with 5R-STS times lower than or equal to the ULN can be considered without relevant functional impairment. Mild functional impairment was graded between the ULN (approximately 50th percentile) and the 75th percentile, whereas moderate and severe functional impairment were between the 76th and 90th and between the 91st and 100th percentiles, respectively.

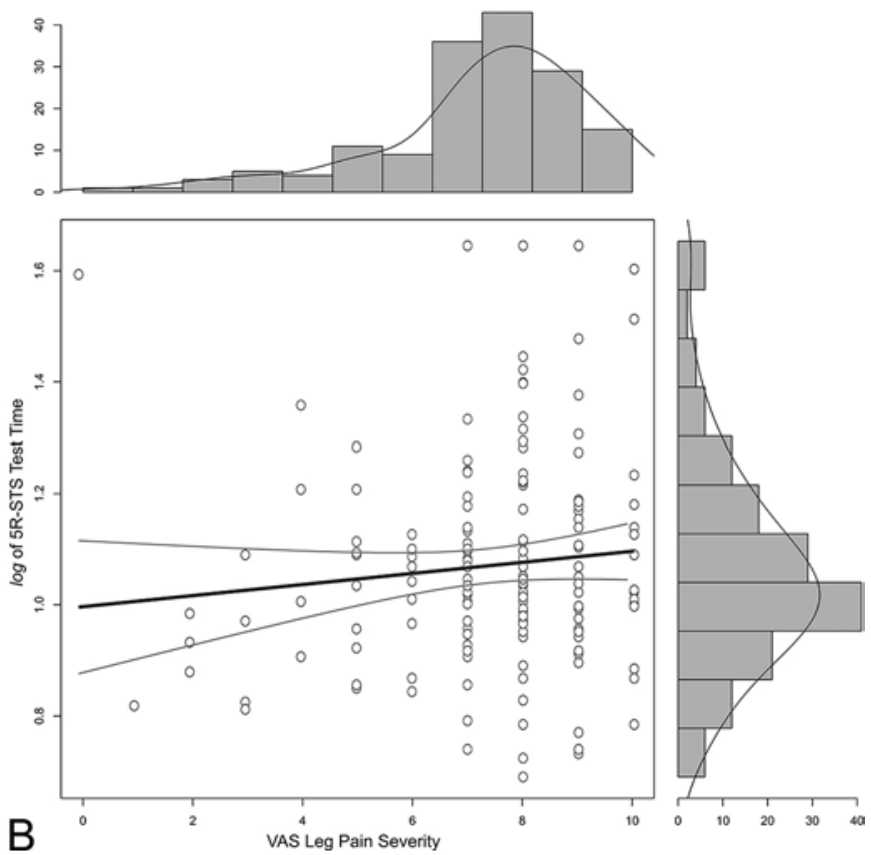

FIG. 3. Scatterplots with marginal histograms showing logarithmically transformed $5 R$-STS test times versus measures of pain severity. There was a direct correlation with the VAS for back pain severity $(\mathbf{A})(r=0.31)$ but not with the VAS for leg pain severity (B) $(r=0.10)$. 

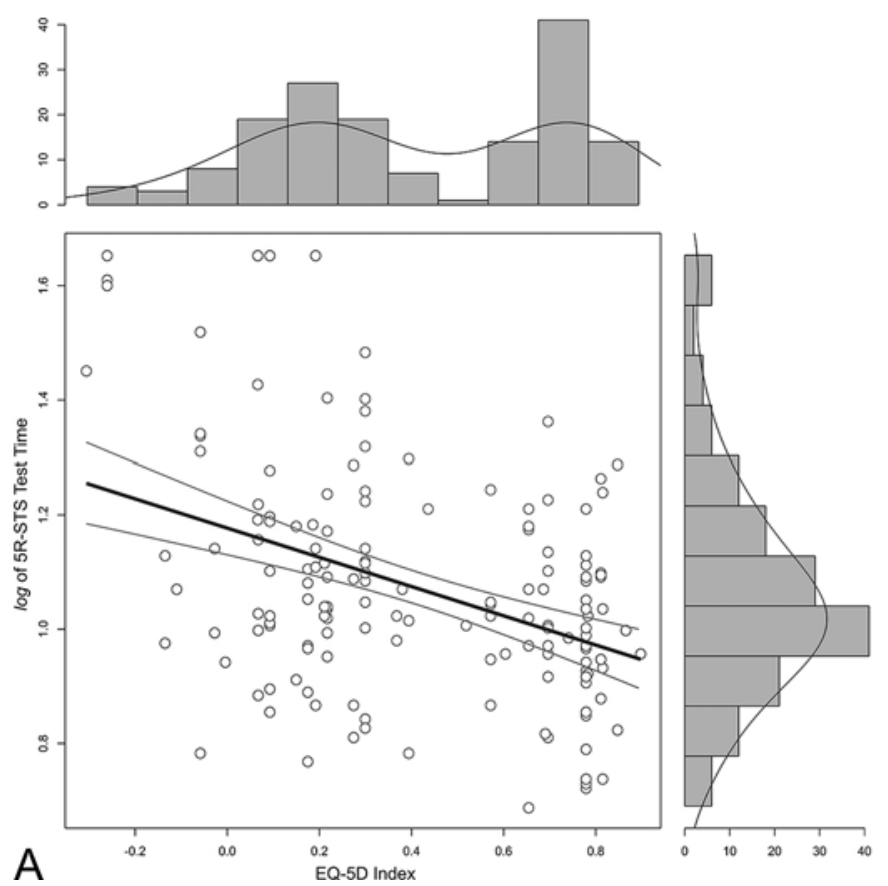

FIG. 4. Scatterplots with marginal histograms showing logarithmically transformed $5 R-S T S$ test times versus measures of $H R Q O L$. There was a direct correlation with the EQ-5D index $(A)(r=-0.41)$ but not with EQ-5D VAS scores $(B)(r=-0.14)$.

\section{Patient-Specific Adjustment}

Age $(\beta=0.037, \mathrm{p}<0.001)$, BMI $(\beta=0.841, \mathrm{p}=0.007)$, height $(\beta=0.249, \mathrm{p}=0.008)$, and weight $(\beta=-0.248$, $\mathrm{p}$ $=0.025$ ) significantly influenced $5 \mathrm{R}$-STS performance in healthy individuals (constant $\left.=-40.9, \mathrm{r}^{2}=0.376\right)$. Sex $(\mathrm{p}=$ $0.14)$ and smoking status $(p=0.37)$ had no effect. The following formula can be used to make a simple prediction of a patient's expected normal test time $\left(t_{a}\right): t_{a}=0.03$ age + $0.15 \mathrm{BMI}+1.7$.

This estimate could be used as targeted 5R-STS performance after successful treatment $\left(r^{2}=0.292\right)$.

\section{Discussion}

In this prospective study, we demonstrated that the 5RSTS test can objectively assess functional impairment in patients with degenerative pathologies of the lumbar spine, with moderate convergent validity and excellent test-retest reliability. Based on our data, we propose an upper limit of normal of 10.4 seconds. This threshold distinguishes between patients with and without relevant functional impairment. The ZOI around this threshold value of 10.4 seconds ranged from 9.3 to 11.4 seconds. In this ZOI, patients cannot unambiguously be determined to have functional impairment or not. Rather, functional impairment in these "gray zone" patients should be judged according to patients' clinical history, or individuals should be reevaluated using a different objective functional test-e.g., the TUG test. ${ }^{9}$ Furthermore, a severity stratification was put in place to help grade test results. Patients can be ranked as having mild, moderate, or severe functional impairment if their 5R-STS test times equate to $10.5-15.2,15.3-22.0$, or greater than 22.0 seconds, respectively. A simple calculation using age and BMI effectively estimates a patient's target 5R-STS test performance.

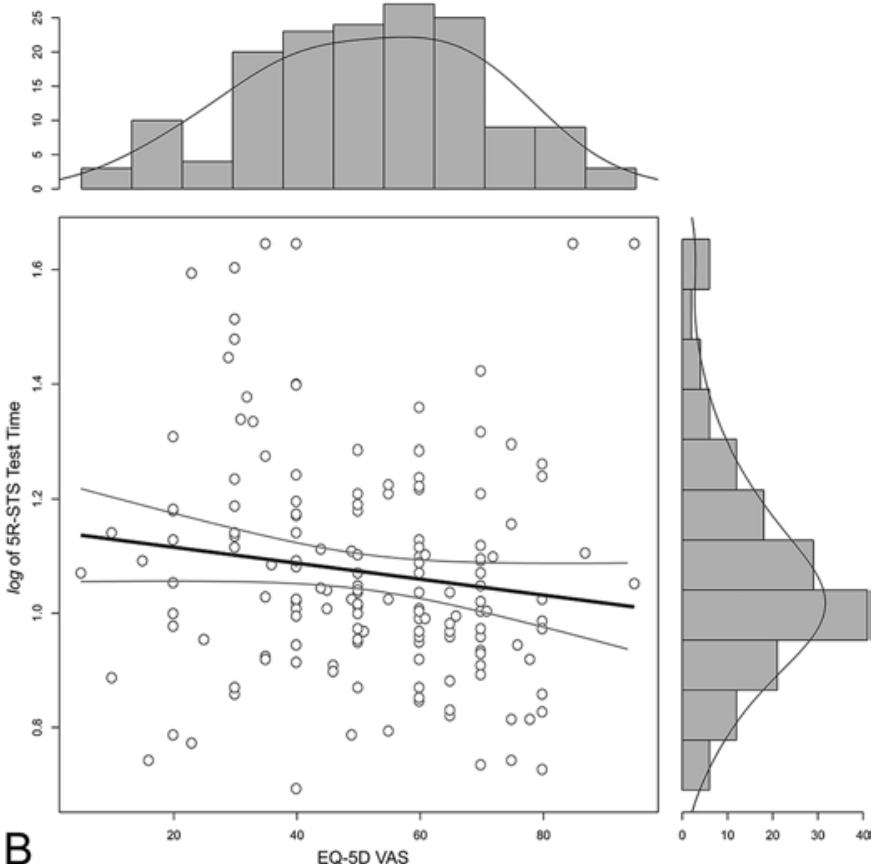

The TUG test has been thoroughly studied in the context of degenerative spinal pathologies. Preoperative and postoperative correlation, the minimum clinically important difference, and patient preference have been assessed, and a baseline severity index has been validated. ${ }^{8-10,24} \mathrm{In}$ this light, the TUG test currently represents the most clinically applicable option for objective functional testing in cases involving lumbar spine surgery. Accordingly, the 5R-STS test does not intend to compete with the TUG test but, rather, to expand the spine surgeon's arsenal for objective functional testing. In some cases, such as when a patient's test result falls within the gray zone of the TUG test, reevaluating the patient with the 5R-STS test, or viceversa, may be appropriate. Surgeons and patients may also prefer one particular test over others for various reasons, ranging from personal inclinations to plain restrictions in space.

The 5R-STS exhibited solid correlation with the RMDQ and ODI as measures of functional impairment, VAS for back pain severity, and the EQ-5D index for HRQOL. Interestingly, there was no consistent correlation with leg pain on the VAS or with the EQ-5D VAS. This indicates that the 5R-STS test is not simply an objectification of pain and pain-related symptoms, which undeniably affect HRQOL, but rather a novel dimension in the assessment of patients, coined "objective functional impairment" (OFI). OFI correlates with, but is not identical to, functional impairment as measured subjectively using PROMs such as the RMDQ and ODI. The excellent correlation between RMDQ and ODI ( $r=0.63)$, as opposed to the good correlation between the 5R-STS test performance and the RMDQ (0.49) or the ODI (0.44), exemplifies this fact.

A patient able to perform the 5R-STS test in $10.4 \mathrm{sec}-$ onds can be considered to have no relevant OFI. Using this 
TABLE 2. Measures of test-retest reliability and measurement error

\begin{tabular}{|c|c|c|c|c|c|c|}
\hline \multirow[b]{2}{*}{ Measure } & \multicolumn{2}{|c|}{ Overall $(n=119)$} & \multicolumn{2}{|c|}{ Study Group $(n=66)$} & \multicolumn{2}{|c|}{ Control Group $(n=53)$} \\
\hline & ICC & $95 \% \mathrm{Cl}$ & ICC & $95 \% \mathrm{Cl}$ & ICC & $95 \% \mathrm{Cl}$ \\
\hline Consistency & 0.98 & $0.97-0.98$ & 0.97 & $0.94-0.98$ & 0.96 & $0.92-0.97$ \\
\hline Absolute agreement & 0.98 & $0.97-0.98$ & 0.97 & $0.94-0.98$ & 0.96 & $0.92-0.97$ \\
\hline SEM & 1.03 & & 1.47 & & 0.34 & \\
\hline
\end{tabular}

threshold value, around half of the patients in our study group were without relevant OFI, as found with the TUG test. ${ }^{9}$ This is of particular interest since all included patients were candidates for surgery with longstanding pain symptoms and failed conservative treatment, demonstrating that OFI is distinctly different from subjective functional impairment and pain, which are frequent indications for surgery. Using a severity stratification (Table 3), the severity of OFI can be graded as mild, moderate, or severe.

Performance on the 5R-STS test is affected by demographic factors such as age, height, weight, and BMI but not by the patient's sex. It has also been shown that seat height confounds 5R-STS performance, underlining the importance of a standardized seat height. ${ }^{19}$ In combination with measurement error, especially those test results that veer close to cutoff values, such as the ULN, must be interpreted cautiously. The emergence of further clinical data in the future will permit the establishment of patientadjusted cutoffs to enhance interpretation.

In the clinical routine, the 5R-STS test is useful in multiple ways. It allows for a simple, quick, and objective estimate of the patient's basic functioning by testing a physical activity that is commonly performed in daily life. As such, it also summarizes the state of the patient's relevant neuromusculature related to the lumbar spinal pathology. These functions are crucial to objectively assess to what extent patients are impaired not only before but also after surgery. They enable the patient to resume activities of daily living and might be an indicator of when patients can be

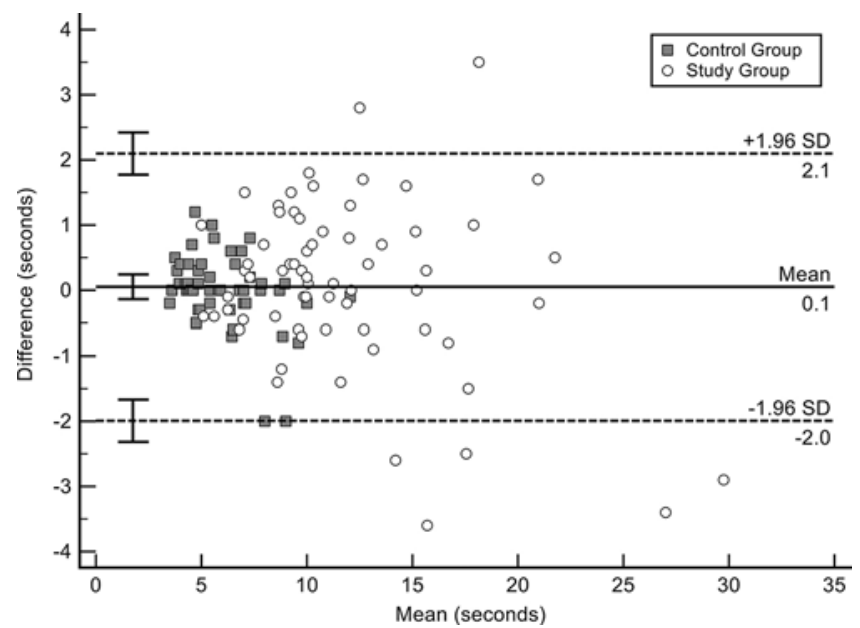

FIG. 5. Bland-Altman plot for test-retest reliability. Mean differences between measurements are plotted against the mean measurements of 5R-STS test times. The interrupted lines represent the $95 \%$ limits of agreement, and the uninterrupted line demonstrates the mean retest bias of 0.1 seconds. discharged home safely. These objective functional tests have been demonstrated to be sensitive to changes after surgical treatment, and this may also be the case for the 5R-STS test. ${ }^{8,10}$ Using simple formulas, we can calculate age- and BMI-adjusted expected test times for any patient to illustrate their current degree of objective functional impairment and to create an expectation of what a positive outcome after surgery might look like. Minor advantages include the fact that the 5R-STS test can be easily administered by physiotherapists and other healthcare personnel and the fact that these tests are not dependent on language, making them suitable for patients who are illiterate. It has been shown that objective functional tests are more robust against the influence of mental health status than a PROM. ${ }^{1,25}$ Moreover, patients prefer performing an objective functional test over completing a battery of PROMs, perhaps because it provides direct and tangible feedback and is less time-consuming. ${ }^{15}$ Considering these factors, the 5R-STS may constitute an excellent follow-up tool that could even be performed by patients themselves at home, either unsupervised or with televised supervision. ${ }^{5}$ It is important to stress that objective functional tests should not replace PROM, since they convey different types of information. Rather, both should be used complementarily in the clinical setting.

\section{Limitations}

The implications of this study may be limited by the sample size. While the present sample size appeared to be sufficient to reach a power of 0.97 according to a post hoc analysis (Appendix), the estimation of correlations relies on large samples, and thus further and larger studies are indicated to give more precise estimates of 5R-STS validity. Since we included patients with various degenerative spinal pathologies to achieve a broad assessment of the 5R-STS test, we cannot make any claims about the validity of this test for each of the specific indications (LDH, stenosis, spondylolisthesis, DDD, and synovial cyst). ${ }^{2}$ However, particularly for $\mathrm{LDH}$, the lesion affecting the majority of patients, the results of this study indicate that there is a strong correlation with the relevant outcome measures that merits further research with larger sample sizes for each specific indication. Confounders that may not have been captured in this study may also potentially influence performance. For example, patient motivation may play a big role in 5R-STS performance. It is to be noted that the control group was younger and included health professionals. As such, our control group may be healthier than the normal population, skewing the ULN. However, most participants in the control group were the patients' partners and exhibited demographics similar to their counterparts. 
TABLE 3. Severity stratification for the 5R-STS test

\begin{tabular}{ccc}
\hline & \multicolumn{2}{c}{ 5R-STS Severity Stratification } \\
\cline { 2 - 3 } Impairment & Test Time $(\mathrm{sec})$ & Prevalence \\
\hline OFI & & \\
\hline Not significant & $\leq 10.4$ & $50 \%$ \\
\hline Mild & $10.5-15.2$ & $25 \%$ \\
\hline Moderate & $15.3-22.0$ & $15 \%$ \\
\hline Severe & $>22.0$ & $10 \%$ \\
\hline
\end{tabular}

Patients' OFI can be graded according to their 5R-STS test time in seconds.

The approximate prevalence of each grade is given.

Last, we have not assessed interrater agreement of the 5RSTS test, which might importantly influence reliability. While there is a need for a specific analysis of interrater agreement in a spinal population, Jones et al. and Mong et al. have demonstrated excellent interrater agreement for the 5R-STS test in different populations. ${ }^{14,18}$ Future studies should focus on validating the 5R-STS test as a follow-up tool and on improved interpretation through the development of highly accurate adjustments, validating the proposed ULN and severity stratification. Of particular clinical relevance is the need to assess the prognostic value of the 5R-STS test overall and in specific degenerative spinal pathologies. While the data presented in the present study are derived from a relatively small sample, we believe that at present they are accurate enough to fundamentally interpret baseline 5R-STS results and to estimate OFI in a clinical setting.

\section{Conclusions}

The 5R-STS test appears to be a valid and reliable measure of OFI. The relevant values for the interpretation of 5R-STS results were determined. Based on our data, we propose an ULN of 10.4 seconds, meaning that patients with a 5R-STS time equal to or lower than this threshold can be considered to be without functional impairment. A severity stratification was also proposed, indicating that patients with test times of 10.5 to $15.2,15.3$ to 22.0 , and greater than 22.0 seconds can be considered to have mild, moderate, and severe OFI, respectively.

\section{Acknowledgments}

We are grateful to all participating volunteers and to Femke Beusekamp, BSc, and Nathalie Schouman for study coordination and data collection. We also thank Marlies P. de Wispelaere, MSc, for her efforts in clinical informatics and Anna M. Nikitin for her artwork.

\section{References}

1. Carreon LY, Djurasovic M, Dimar JR II, Owens RK II, Crawford CH III, Puno RM, et al: Can the anxiety domain of EQ-5D and mental health items from SF-36 help predict outcomes after surgery for lumbar degenerative disorders? J Neurosurg Spine 25:352-356, 2016

2. Crawford CH III, Carreon LY, Bydon M, Asher AL, Glassman SD: Impact of preoperative diagnosis on patient satisfaction following lumbar spine surgery. J Neurosurg Spine 26:709-715, 2017
3. Deyo RAM, Battie M, Beurskens AJHM, Bombardier C, Croft P, Koes B, et al: Outcome measures for low back pain research. A proposal for standardized use. Spine (Phila Pa 1976) 23:2003-2013, 1998

4. Duncan RP, Leddy AL, Earhart GM: Five times sit-to-stand test performance in Parkinson's disease. Arch Phys Med Rehabil 92:1431-1436, 2011

5. Ejupi A, Brodie M, Gschwind YJ, Lord SR, Zagler WL, Delbaere K: Kinect-based five-times-sit-to-stand test for clinical and in-home assessment of fall risk in older people. Gerontology 62:118-124, 2015

6. Fairbank JC, Couper J, Davies JB, O'Brien JP: The Oswestry low back pain disability questionnaire. Physiotherapy 66:271-273, 1980

7. Gautschi OP, Corniola MV, Schaller K, Smoll NR, Stienen $\mathrm{MN}$ : The need for an objective outcome measurement in spine surgery - the timed-up-and-go test. Spine J 14:25212522, 2014

8. Gautschi OP, Joswig H, Corniola MV, Smoll NR, Schaller $\mathrm{K}$, Hildebrandt G, et al: Pre- and postoperative correlation of patient-reported outcome measures with standardized Timed Up and Go (TUG) test results in lumbar degenerative disc disease. Acta Neurochir (Wien) 158:1875-1881, 2016

9. Gautschi OP, Smoll NR, Corniola MV, Joswig H, Chau I, Hildebrandt G, et al: Validity and reliability of a measurement of objective functional impairment in lumbar degenerative disc disease: the timed up and go (TUG) test. Neurosurgery 79:270-278, 2016

10. Gautschi OP, Stienen MN, Corniola MV, Joswig H, Schaller $\mathrm{K}$, Hildebrandt G, et al: Assessment of the minimum clinically important difference in the timed up and go test after surgery for lumbar degenerative disc disease. Neurosurgery 80:380-385, 2017

11. Guyatt GH, Sullivan MJ, Thompson PJ, Fallen EL, Pugsley SO, Taylor DW, et al: The 6-minute walk: a new measure of exercise capacity in patients with chronic heart failure. Can Med Assoc J 132:919-923, 1985

12. Gvozdyev BV, Carreon LY, Graves CM, Riley SA, McGraw KE, Head RJ, et al: Patient-reported outcome scores underestimate the impact of major complications in patients undergoing spine surgery for degenerative conditions. J Neurosurg Spine 27:397-402, 2017

13. Hoeymans N, van Lindert H, Westert GP: The health status of the Dutch population as assessed by the EQ-6D. Qual Life Res 14:655-663, 2005

14. Jones SE, Kon SSC, Canavan JL, Patel MS, Clark AL, Nolan $\mathrm{CM}$, et al: The five-repetition sit-to-stand test as a functional outcome measure in COPD. Thorax 68:1015-1020, 2013

15. Joswig H, Stienen MN, Smoll NR, Corniola MV, Chau I, Schaller K, et al: Patients' preference of the timed up and go test or patient-reported outcome measures before and after surgery for lumbar degenerative disk disease. World Neurosurg 99:26-30, 2017

16. Landis JR, Koch GG: The measurement of observer agreement for categorical data. Biometrics 33:159-174, 1977

17. Mobbs RJ, Phan K, Maharaj M, Rao PJ: Physical activity measured with accelerometer and self-rated disability in lumbar spine surgery: a prospective study. Global Spine J 6:459-464, 2016

18. Mong Y, Teo TW, Ng SS: 5-repetition sit-to-stand test in subjects with chronic stroke: reliability and validity. Arch Phys Med Rehabil 91:407-413, 2010

19. Ng SSM, Cheung SY, Lai LSW, Liu ASL, Ieong SHI, Fong SSM: Association of seat height and arm position on the five times sit-to-stand test times of stroke survivors. BioMed Res Int 2013:642362, 2013

20. Porchet F, Lattig F, Grob D, Kleinstueck FS, Jeszenszky D, Paus C, et al: Comparison of patient and surgeon ratings of outcome 12 months after spine surgery: presented at the 2009 
Joint Spine Section Meeting. J Neurosurg Spine 12:447455, 2010

21. Rabin R, de Charro F: EQ-5D: a measure of health status from the EuroQol Group. Ann Med 33:337-343, 2001

22. Roland M, Morris R: A study of the natural history of back pain. Part I: development of a reliable and sensitive measure of disability in low-back pain. Spine (Phila Pa 1976) 8:141144, 1983

23. Sánchez-Zuriaga D, López-Pascual J, Garrido-Jaén D, de Moya MFP, Prat-Pastor J: Reliability and validity of a new objective tool for low back pain functional assessment. Spine (Phila Pa 1976) 36:1279-1288, 2011

24. Stienen MN, Smoll NR, Joswig H, Corniola MV, Schaller $\mathrm{K}$, Hildebrandt G, et al: Validation of the baseline severity stratification of objective functional impairment in lumbar degenerative disc disease. J Neurosurg Spine 26:598-604, 2017

25. Stienen MN, Smoll NR, Joswig H, Snagowski J, Corniola MV, Schaller K, et al: Influence of the mental health status on a new measure of objective functional impairment in lumbar degenerative disc disease. Spine J 17:807-813, 2017

26. Stratford PW, Goldsmith CH: Use of the standard error as a reliability index of interest: an applied example using elbow flexor strength data. Phys Ther 77:745-750, 1997

\section{Disclosures}

The authors report no conflict of interest concerning the materi- als or methods used in this study or the findings specified in this paper.

\section{Author Contributions}

Conception and design: both authors. Acquisition of data: both authors. Analysis and interpretation of data: both authors. Drafting the article: Staartjes. Critically revising the article: both authors. Reviewed submitted version of manuscript: both authors. Approved the final version of the manuscript on behalf of both authors: Staartjes. Statistical analysis: both authors. Administrative/technical/material support: Schröder. Study supervision: both authors.

\section{Supplemental Information \\ Online-Only Content}

Supplemental material is available with the online version of the article.

Appendix. https://thejns.org/doi/suppl/10.3171/2018.2.

\section{SPINE171416.}

\section{Correspondence}

Victor E. Staartjes: Bergman Clinics, Naarden, The Netherlands. victor.staartjes@gmail.com. 